\title{
Umsetzung Betrieblicher Gesundheitsförderung/Betrieblichen Gesundheitsmanagements in Deutschland: Stand und Entwicklungsbedarfe der einschlägigen Forschung
} Implementation of Workplace Health Promotion/Workplace Health Management in Germany: State-of-the Art and Need for Further Research

() (1) $(9$

\author{
Autor \\ G. Faller
}

Institut

Department of Community Health, Hochschule für Gesundheit Bochum, Bochum

\section{Schlüsselwörter}

Gesundheitsförderung, Gesundheitsmanagement, Management, Betrieb, Arbeitsplatz

Key words

health promotion, health management, management, organisational development, workplace

Korrespondenzadresse

Prof. Gudrun Faller

Department of Community Health

Hochschule für Gesundheit Bochum

Universitätsstraße 105

44789 Bochum

gudrun.faller@hs-gesundheit.de

\section{Bibliografie}

DOI http://dx.doi.org/10.1055/s-0042-100624

Online-Publikation: 23.3.2016

Gesundheitswesen 2018; 80: 278-285

(c) Georg Thieme Verlag KG Stuttgart · New York ISSN 0941-3790

\section{ZUSAMMENFASSUNG}

Ziel der Studie Zur Frage der Verbreitung von Gesundheitsförderung/Gesundheitsmanagement in deutschen Betrieben liegen heterogene Ergebnisse vor. Das Ziel der Studie besteht darin, mehr Transparenz über die Ursachen dieser Diskrepanzen sowie zu möglichen Einflussfaktoren auf die Bereitschaft zur Umsetzung von BGF/BGM zu gewinnen.

Methodik Auf Basis einer systematischen Datenbankrecherche wurden einschlägige Studien identifiziert und einer vergleichenden inhaltlichen Analyse unterzogen.

Ergebnisse Die Studien zur Verbreitung von BGF/BGM in Deutschland weisen hinsichtlich Fragestellung, Repräsentati- vität, Vorgehen und Design erhebliche Unterschiede auf. Dennoch zeichnen sich übereinstimmende Tendenzen hinsichtlich der Identifikation signifikanter Einflussfaktoren auf die Bereitschaft zur Umsetzung von BGF/BGM ab.

Schlussfolgerungen Bei der Konzeption künftiger Studien zur Analyse der Verbreitung von BGF/BGM ist es wichtig, sich auf eine einheitliche Definition sowie ein vergleichbares Erfassungsund Kategorisierungsschema der erhobenen Maßnahmen zu einigen. Im Hinblick auf die Auswertung von Auswirkungen der betrieblichen Interventionen wären die quantitativen durch qualitative Daten zu ergänzen, die in der Lage sind, das „Wie“ der Umsetzung sowie darauf bezogene, spezifische organisationskulturelle Deutungsmuster zu erfassen.

\section{ABSTRACT}

Aim The data on health management programmes in German companies show quite heterogeneous results. The aim of the study was to gain more transparency into the cause of these discrepancies as well as information on factors influencing the willingness of companies to implement such programmes.

Methods Based on a systematic database search, relevant studies were identified and comparative analysis of their contents was carried out.

Results The existing studies on the health management programmes in German companies reveal significant discrepancies in terms of their research question, representativeness, approach and design. Nevertheless, they do reveal certain trends identifying significant factors that influence the willingness of companies to implement such programmes.

Conclusion In the design of future studies to analyse the distribution of corporate health management programmes, there is a need to agree on common definitions and data collection methods and how the results are to be categorised. When evaluating the effects of corporate programmes, the quantitative data would need to be supplemented with qualitative data that would be capable of identifying how such programmes are implemented and the associated organisation-specific cultural patterns. 


\section{Fragestellungen}

BGF/BGM gelten in Deutschland als sehr erfolgreiche Ansätze lebensweltbezogener Gesundheitsförderung. Allein der aktuelle Präventionsbericht des Medizinischen Dienstes des Spitzenverbandes Bund der Krankenkassen bescheinigt dem Setting Betrieb 68\% aller Ausgaben im nicht-individualpräventiven Bereich [1]. Zudem hat die gesundheitsökonomische Forschung der BGF bzw. dem BGM in den letzten Jahren deutliche Nutzeneffekte und Effizienzgewinne zugesprochen [2-4]. Daher erscheint es naheliegend, wenn Unternehmer aus ökonomischen Erwägungen ebenso wie aus Gründen einer gelebten Unternehmerverantwortung und eines zeitgemäßen Arbeits- und Gesundheitsschutzes gesundheitsfördernde Programme implementieren [5]. Ob dem so ist, wurde in der Vergangenheit im Rahmen von diversen Verbreitungsstudien ermittelt. Ziel der vorliegenden Analyse ist es, aus einer vergleichenden Gegenüberstellung dieser Arbeiten zu ermitteln

1. welche Aussagen zu potenziell begünstigenden bzw. behin-

dernden Einflussgrößen auf die Umsetzung von BGF/BGM

abgeleitet werden können

2. auf welche methodischen Faktoren die unterschiedlichen

Aussagen zur prozentualen Verbreitung von BGF/BGM in

Deutschland zurückzuführen sind

Aus den Ergebnissen werden Anforderungen an Konzeption und Design künftiger Verbreitungsstudien abgeleitet sowie weitergehende Überlegungen zur Erfassung der Wirkungen der Umsetzung von BGF angestellt.

\section{Zum Begriffsverständnis von BGF und BGM}

Das European Network for Workplace Health Promotion (ENWHP) versteht unter Betrieblicher Gesundheitsförderung (BGF) „alle gemeinsamen Maßnahmen von Arbeitgebern, Arbeitnehmern und Gesellschaft zur Verbesserung von Gesundheit und Wohlbefinden am Arbeitsplatz“ [6]. Als konkrete Interventionen nennt die hier zitierte Luxemburger Deklaration „Verbesserung der Arbeitsorganisation und der Arbeitsbedingungen“, „Förderung einer aktiven Mitarbeiterbeteiligung“ und die „Stärkung persönlicher Kompetenzen“. BGF versteht sich als ein Gesamtkonzept, das gesundheitsfördernde Unternehmensgrundsätze, Unternehmenskulturen und Führungsleitlinien enthält, Wert auf die Beteiligung und Förderung der Beschäftigten legt und dabei den Arbeitsschutz integriert. Badura et al. [7] definieren Betrieblichem Gesundheitsmanagement (BGM) als „die Entwicklung integrierter betrieblicher Strukturen und Prozesse, die die gesundheitsförderliche Gestaltung von Arbeit, Organisation und dem Verhalten am Arbeitsplatz zum Ziel haben und den Beschäftigten wie dem Unternehmen gleichermaßen zugute kommen “(S. 17). In beiden Quellen wird die Relevanz der Gestaltung der betrieblichen Rahmenbedingungen betont, die Notwendigkeit eines systematischen Vorgehens, die Verantwortung des Managements und die Bedeutung der Einbeziehung der Beschäftigten. Aufgrund der in der Praxis verbreiteten oft synonymen Verwendung und, um ein breites Spektrum von Aktivitäten zu erfassen, liegt diesem Beitrag eine parallele Nutzung der Begriffe ,Betriebliche Gesundheitsförderung (BGF) wie auch ,Betriebliches Gesundheitsmanagement' (BGM) zugrunde.

\section{Methodik}

Zunächst wurde eine systematische Datenbankrecherche von einschlägigen empirischen Arbeiten mit Veröffentlichungsdatum seit dem Jahr 2000 durchgeführt. Die Recherche wurde in deutscher und englischer Sprache realisiert. Als Suchbegriffe wurde folgende Kombination verwendet: ((Betriebliche * AND Gesundheits *) OR „Workplace Health Promotion“ OR BGF OR BGM) AND (implement * OR survey OR dissemination OR spread * OR Deutschland OR Germany OR Verbreitung OR frequency OR Häufigkeit). Herangezogen wurden die Datenbanken PubMed, PubPsych inklusive PPSYNDEX, PASCAL, ISOC-Psicologia, MEDLINE, ERIC, NARCIS, NORART, PsychOpen und PsychData, weiter PPSYNDEX, PsychINFO und SocINDEX über Cinahl. Von den aufgrund der Titelformulierungen in Frage kommenden Auswahlen wurden zunächst die deutsch- bzw. englischsprachigen Abstracts, in Zweifelsfällen die Volltexte gesichtet. Nach Aussonderung nicht geeigneter Treffer und Streichung von Doppelungen fanden insgesamt 10 Literaturquellen im Rahmen der Datenbankrecherche Berücksichtigung. Als ergänzende Quellen wurden die in den einschlägigen Treffern enthaltenen Verweise auf verwandte Studien berücksichtigt, sowie das im Rahmen der langjährigen fachlichen Beschäftigung mit der Thematik angelegte Facharchiv der Autorin. Auf diese Weise konnte die Auswahl um weitere 6 Quellen erweitert werden. Abschließend fanden insgesamt 16 Literaturquellen im Rahmen der Datenbankrecherche Berücksichtigung, diese beziehen sich auf 13 Analysen. Als Auswahlkriterien dienten folgende Anforderungen an die Studien:

1. Unternehmensbezug: Es wurden nur Studien einbezogen, welche Unternehmer bzw. betriebliche Experten als Adressaten ansprachen. Arbeiten, die eine Verbreitung von Gesundheitsförderung aus der Perspektive der Beschäftigten darstellen, oder bei denen der Unternehmensbezug aus anderen Gründen nicht deutlich wurde, waren dagegen ausgeschlossen.

2. Umfassendes Verständnis von Gesundheitsförderung: Exkludiert wurden Untersuchungen, die sich auf spezifische Themen konzentrierten (z. B. ausschließlich Maßnahmen zur Umsetzung der Gefährdungsbeurteilung psychischer Belastungen, der Suchtprävention, der betrieblichen Wiedereingliederung usw.).

3. Angaben zur prozentualen Verbreitung: Keine Berücksichtigung fanden Analysen, die zwar betriebliche Gesundheitsmaßnahmen erhoben, diese in ihren Ergebnisdarstellungen jedoch nicht als \%-Werte sondern ausschließlich in Form von Indexwerten auswiesen.

\section{Ergebnisse}

- Tab. 1 zeigt die im Ergebnis ermittelten Studien seit dem Jahr 2000. Publikationen, die dieselbe Studie beschreiben, sind jeweils in einer Zeile aufgeführt.

\section{A) Einflussfaktoren auf die Umsetzung von BGF/BGM}

Die berücksichtigten Studien setzen sich in unterschiedlichem Umfang mit der Frage auseinander, welche Merkmale in einem kausalen Zusammenhang mit der Realisierungsbereitschaft zu BGF/BGM stehen. Dabei werden verschiedene Faktoren im Hinblick auf mögliche Korrelationen untersucht. 


\begin{tabular}{|c|c|c|c|c|c|c|c|c|c|}
\hline 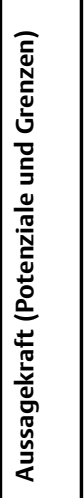 & 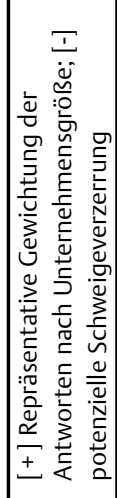 & 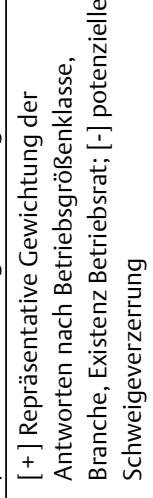 & 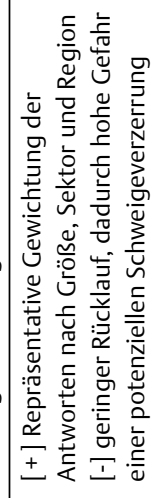 & 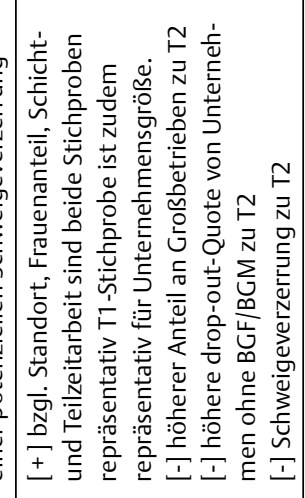 & 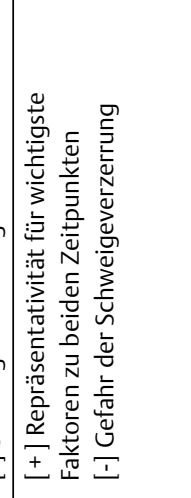 & 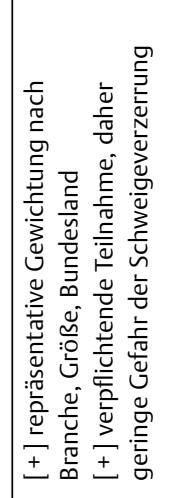 & 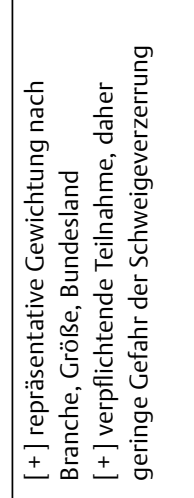 & 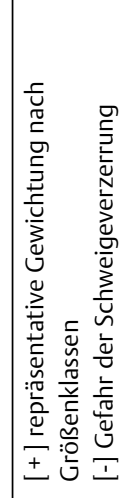 & 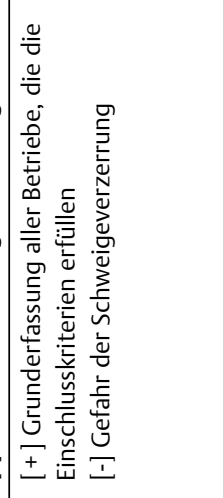 \\
\hline 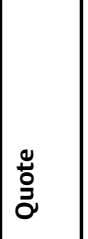 & 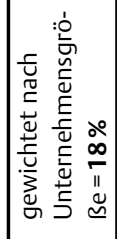 & 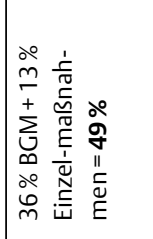 & 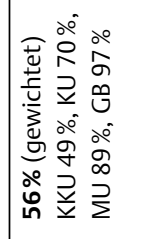 & $\begin{array}{l}\stackrel{\circ}{0} \\
\infty \\
\ddot{\tilde{N}} \\
\stackrel{0}{0} \\
\infty \\
\ddot{F}\end{array}$ & $\begin{array}{l}\stackrel{\circ}{\tilde{N}} \\
\dot{\tilde{N}} \\
\stackrel{\circ}{ } \\
\dddot{m} \\
\ddot{F}\end{array}$ & 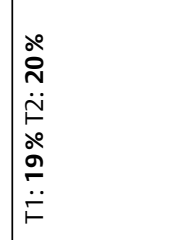 & $\stackrel{\circ}{\stackrel{\circ}{~}}$ & ஓ̊ & 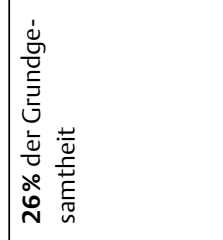 \\
\hline 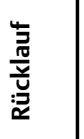 & $\frac{\grave{N}}{\grave{N}}$ & $\stackrel{\dot{\Xi}}{\dot{\Sigma}}$ & 우 & $\begin{array}{ll}\circ & \circ \\
00 & 0 \\
0 & 0 \\
\ddot{F} & \ddot{\sim}\end{array}$ & 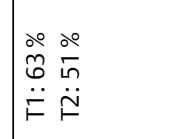 & 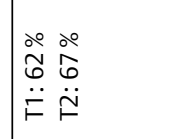 & $\stackrel{\dot{\Sigma}}{\dot{\Sigma}}$ & $\stackrel{\circ}{\grave{N}}$ & 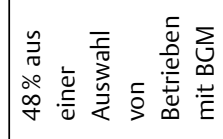 \\
\hline 它 & ڤે & ஓे & $\bar{i}$ & 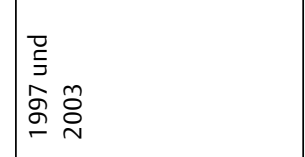 & 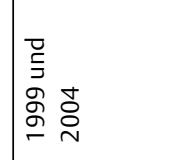 & 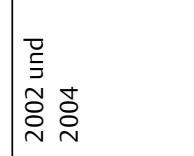 & 完 & 㐫 & 总 \\
\hline & 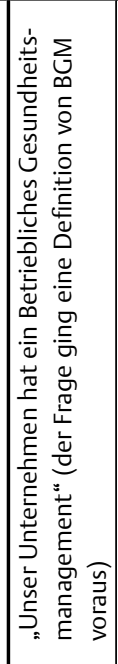 & 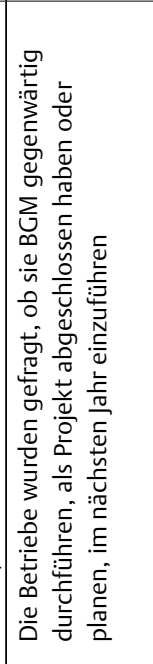 & 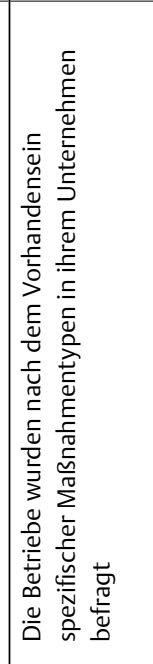 & 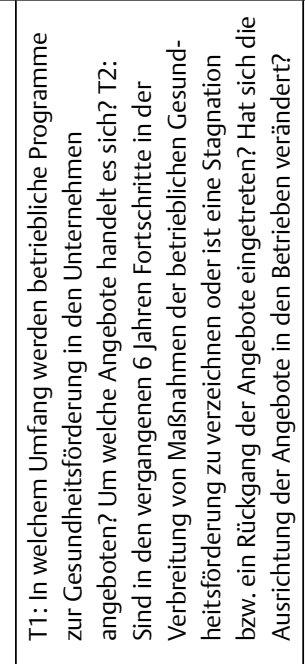 & 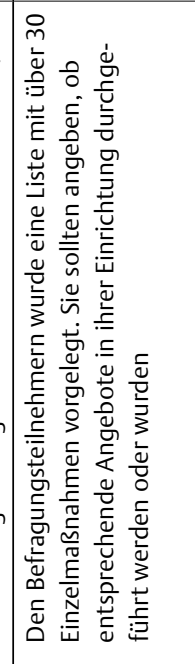 & 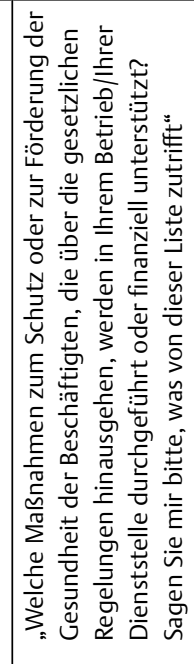 & 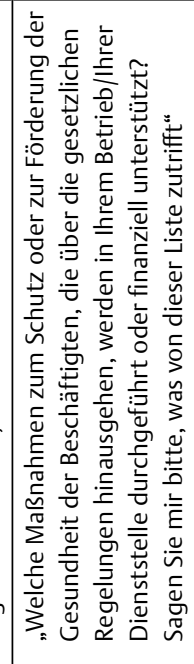 & 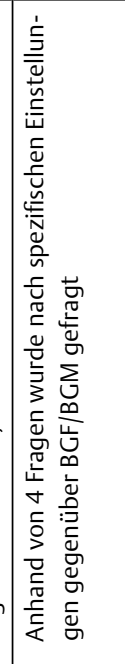 & 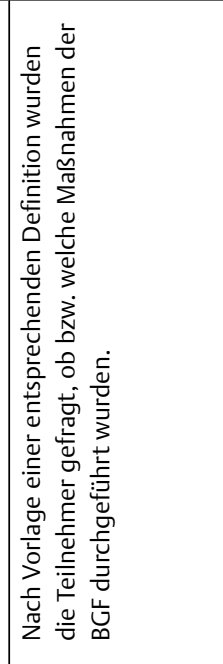 \\
\hline 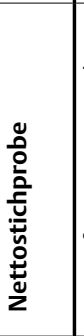 & 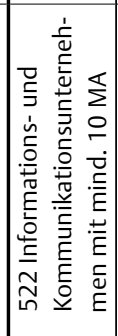 & 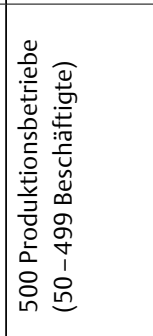 & 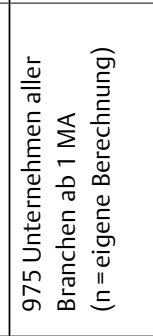 & 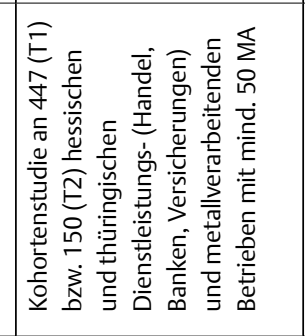 & 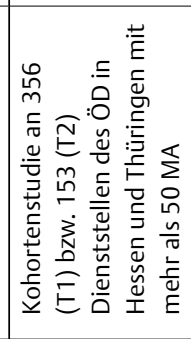 & 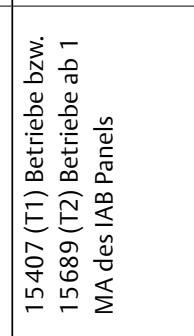 & 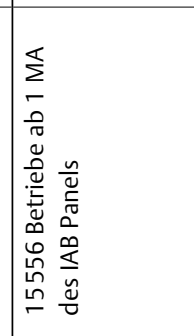 & 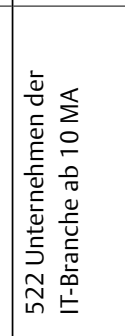 & 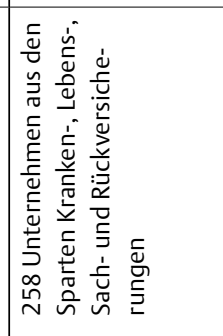 \\
\hline 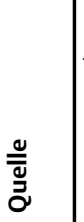 & 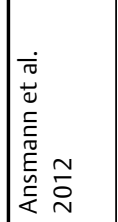 & 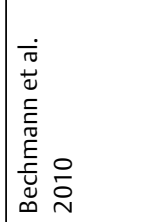 & 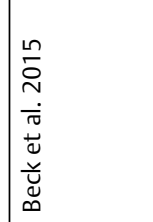 & 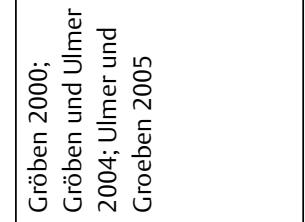 & 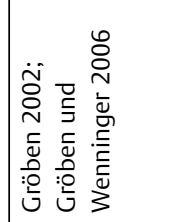 & 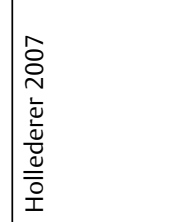 & 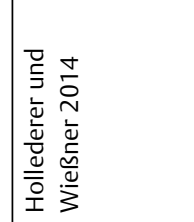 & 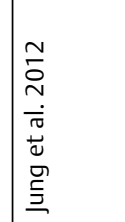 & 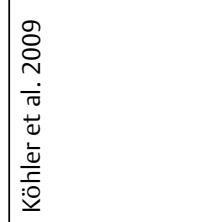 \\
\hline
\end{tabular}




\begin{tabular}{|c|c|c|c|c|c|}
\hline 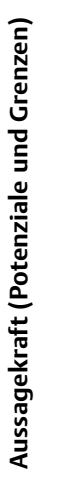 & 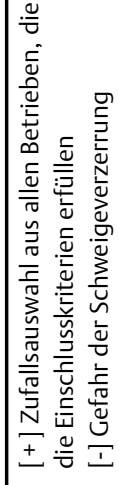 & 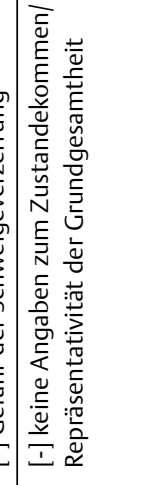 & 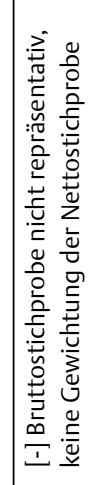 & 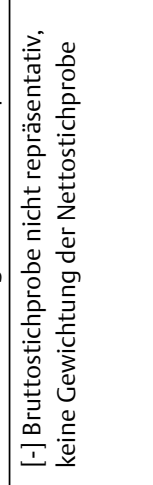 & 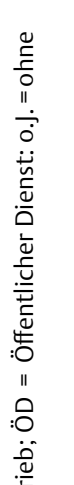 \\
\hline 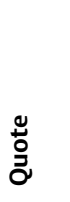 & 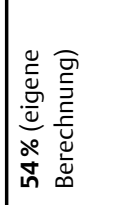 & ஃ̊ & 哭 & $\stackrel{\stackrel{一}{m}}{m}$ & 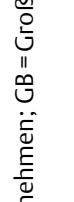 \\
\hline 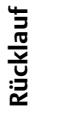 & $\begin{array}{l}\stackrel{\circ}{i} \\
\text { in }\end{array}$ & 离 & $\begin{array}{l}\stackrel{\circ}{\circ} \\
\stackrel{\infty}{+}\end{array}$ & $\stackrel{\dot{\Sigma}}{\dot{\Sigma}}$ & 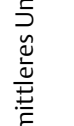 \\
\hline 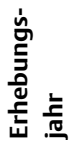 & ঃ & $\stackrel{\sim}{\stackrel{\sim}{\sim}}$ & ఫ্ণ & $\stackrel{\infty}{\stackrel{\sim}{~}}$ & 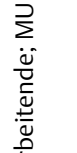 \\
\hline 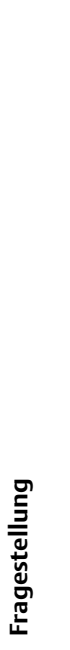 & 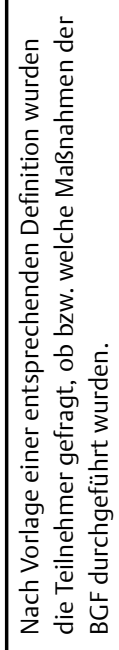 & 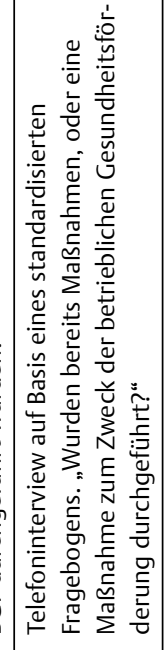 & 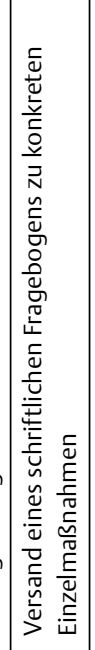 & 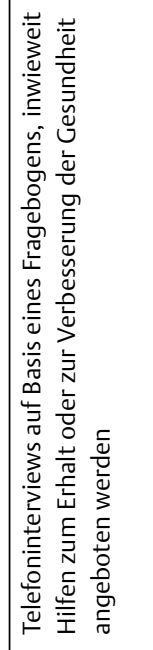 & 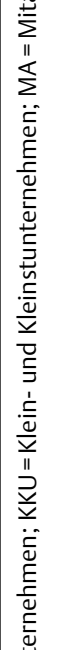 \\
\hline 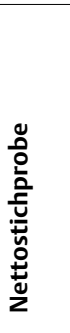 & 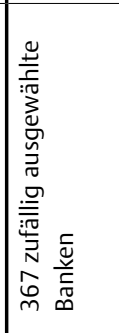 & 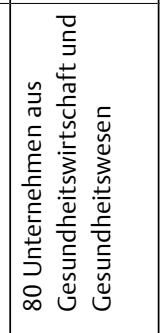 & 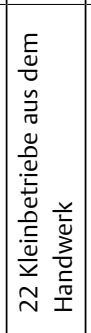 & 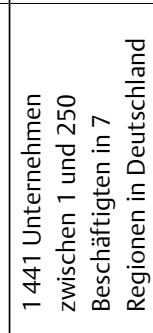 & 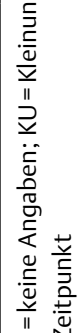 \\
\hline $\begin{array}{l}\frac{\mathscr{\varrho}}{\widetilde{\Xi}} \\
\text { }\end{array}$ & 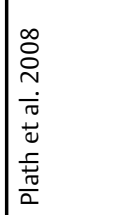 & 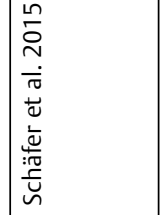 & 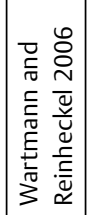 & 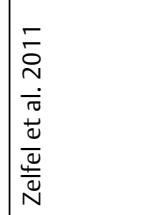 & 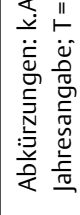 \\
\hline
\end{tabular}

Die größte Einigkeit besteht für den Faktor „Betriebsgröße“. Hier lässt sich zusammenfassend konstatieren, dass je größer der Betrieb ist, umso eher Maßnahmen der/des BGF/BGM umgesetzt werden. Diesen Zusammenhang bestätigen 11 der insgesamt 13 einbezogenen Studien. Ansmann et al. [8] benennen für die von ihnen analysierten IT-Unternehmen ein adjustiertes Odds Ratio (OR) von 1,5 (nicht signifikant) für Mittelbetriebe (50-249 Beschäftigte) und von 2,8 (signifikant) für Betriebe ab 250 Mitarbeitenden im Vergleich zu Betrieben unter 50 Personen. Hollederer und Wießner [9] beschreiben bei Kontrolle aller identifizierten, anderen signifikanten Einflussfaktoren eine Erhöhung der Wahrscheinlichkeit mindestens eine Maßnahme zu realisieren von $15 \%$ für Kleinbetriebe (20-49 Mitarbeitende), von 31 \% für mittlere Betriebe (50-499 MA) und von 35\% für Großbetriebe (ab 500 MA) - jeweils im Vergleich zu Kleinstbetrieben (5-19 MA).

Heterogener sind die Ergebnisse dagegen für das Merkmal „Branche“. Zwar führen mehrere Arbeiten sektorenbezogene Vergleiche auf, z. B. [9-12]; wegen unterschiedlicher Selektionen und Zuordnungen sind übereinstimmende Aussagen jedoch schwer zu treffen. Aufgrund der großen Stichprobe und des hohen Rücklaufs dürfte den IAB-Paneldatenauswertungen dabei eine prominente Bedeutung zukommen [9, 12]. Diese Daten weisen eine überdurchschnittliche Verwirklichungsquote für den Öffentlichen Dienst aus, während etwa das Gastgewerbe eine Schlussposition einnimmt. Innerhalb des produzierenden Gewerbes beschreiben Bechmann et al. [10] Differenzen für Betriebe mittlerer Größe, wobei die Produktionsgüterindustrie einige Prozentpunkte vor der Nahrungsund Genussmittelproduktion rangiert. Der Effekt der Branche zeigte sich in der von Hollederer and Wießner [9] modellierten Regression insbesondere für den Öffentlichen Dienst, selbst bei Kontrolle anderer Einflussgrößen. Der Dienstleistungssektor weist im Vergleich zum Produktionssektor eine um 2 \% erhöhte Umsetzungswahrscheinlichkeit mindestens einer Maßnahme auf.

Einen mit relativ hoher Übereinstimmung abgesicherten Einfluss übt das Vorhandensein einer Beschäftigtenvertretung aus. Insgesamt 5 Studien untersuchen diesen Zusammenhang und bestätigen ihn [8-12]. Bechmann et al. [10] bemerken, dass dieser Effekt auch innerhalb der 3 Betriebsgrößengruppen auftritt. Ansmann et al. [8] dagegen konnten in ihrer Erhebung einen signifikanten Effekt nur für Kleinbetriebe ausmachen, und bei Jung et al. [13] verschwand der Effekt nach Kontrolle der anderen Einflussfaktoren. Ansmann et al. [8] berechnen für Kleinbetriebe ein OR von 2,5 im Vergleich zu Betrieben ohne Beschäftigtenvertretung. Im Regressionsmodell von Hollederer and Wießner [9], das mehrere relevante Einflussgrößen kontrolliert, zeigen gewerbliche Betriebe mit einer Beschäftigtenvertretung eine nahezu 21 \% ig höhere Umsetzungswahrscheinlichkeit.

Ob der regionale Standort für die Umsetzung von BGF eine Rolle spielt, dazu gehen die Resultate auseinander. Während Zelfel et al. [14] für ihre - allerdings nicht repräsentative - Stichprobe keinen Einfluss ermitteln konnten, finden andere Studien übereinstimmend eine höhere Umsetzungsquote in den neuen Bundesländern $[10,12]$ bzw. ein höheres Qualitätsniveau der BGF [15]. Letzeres wurde nach Gröben und Ulmer jedoch nur für den öffentlichen Dienst gefunden, nicht für gewerbliche Betriebe [16]. Für die aktuelle Wiederholungsbefragung im Rahmen des IAB-Panels räumen Hollederer and Wießner [9] eine Annäherung zwischen Ost und 
West, und bei Kontrolle aller anderen Faktoren nur noch eine gering erhöhte Verbreitungswahrscheinlichkeit von $3 \%$ ein.

Als weitere korrelierende Größen wurden das Vorhandensein einer Fachkraft für Arbeitssicherheit [11], eines Gesundheitskoordinators [11] und die Präsenz eines Betriebsarztes [8] gefunden, wobei der Effekt in letzterem Fall bei einer Korrektur durch die Unternehmensgröße verschwindet. Des Weiteren ließen sich erwartete Probleme bei der Personalrekrutierung [9] als fördernde Faktoren verifizieren. Für den Einfluss des wirtschaftlichen Drucks liegen unterschiedliche Ergebnisse vor: Während Köhler et al. [17] diesen als fördernden Faktor bestätigen konnten, sprechen Hollederer und Wießner [9] dem wirtschaftlichen Druck keinen Einfluss auf die Wahrscheinlichkeit der Umsetzung von BGF/BGM zu. Im Gegensatz dazu konnten einige Autoren [13] eine bevorzugte Marktposition als positiven Einflussfaktor ausmachen. Jung et al. [13] identifizierten die Zahl der Hierarchieebenen, die Anzahl der akademisch qualifizierten Beschäftigten und den Anteil an unbefristeten Arbeitsverhältnissen als eigenständige positive Einflussfaktoren. Wenig überraschend ist die Beobachtung, dass Unternehmen mit systematischen Konzepten in anderen Bereichen der Personal- und Organisationsentwicklung [17] bzw. mit einem hohen Qualitätsbewusstsein [18], einer ausgeprägten Wertschätzung des Arbeits- und Gesundheitsschutzes [16, 19] und umfassender Inanspruchnahme von Beratung durch externe Stellen [19] gleichzeitig eine stärkere Performance im Bereich BGF/BGM aufweisen. An den Untersuchungen der Arbeitsgruppe um Gröben fällt auf, dass einzelne Einflussfaktoren auf die Qualität von BGF nur für den öffentlichen Dienst bestätigt werden konnten, nicht aber für die gewerbliche Wirtschaft. Dazu zählen die Kenntnis der Fehlzeitenquote, das Ausmaß der Belastungen bei den Beschäftigten, vorliegende Berufskrankheitenanzeigen und das Ausmaß der Belastungen in der Belegschaft [16, 19]. Demnach sind bestimmte Einflussfaktoren nur in spezifischen Bereichen oder Sektoren wirksam. Die hier in Einzelstudien untersuchten Faktoren sollten durch die weitere Forschung näher analysiert und auf Wechselwirkungen hin überprüft werden.

\section{B) Einflüsse der Erhebungsmethodik auf die ermittelte Umsetzungsquote}

Im Hinblick auf die Umsetzungsquoten fällt die breite Varianz von $18 \%$ bis zu $93 \%$ auf. Aus der tiefergehenden Auseinandersetzung ergaben sich folgende, dem Untersuchungsdesign geschuldete Ursachen:

Stichprobenzusammensetzung Von den 12 in die Auswertung einbezogenen Untersuchungen bemühten sich insgesamt 9 um die Anforderungen an Vergleichbarkeit mit der jeweiligen Grundgesamtheit - teilweise durch eine repräsentative Auswahl der Befragungsteilnehmer und/oder teils durch die nachträgliche Gewichtung der Ergebnisse. Dabei wurde meistens der Faktor Betriebsgröße berücksichtigt, in den Fällen fanden noch weitere Strukturmerkmale Eingang in die Gewichtung ( $\vee$ Tab. 1). Einschränkend gilt jedoch, dass selbst bei den nachträglich nach Betriebsgröße gewichtenden Arbeiten oftmals Kleinst- und/oder Kleinbetriebe ausgenommen waren. Aufgrund des oben beschriebenen Einflusses der Unternehmensgröße ist davon auszugehen, dass die tatsächliche Umsetzungsquote in diesen Fällen geringer sein dürfte, als die in den Studien ermittelte. Von den als repräsentativ geltenden Arbeiten beziehen sich nur $3[9,11,12]$ auf die gesamte deutsche Wirtschaft, während die übrigen spezifische Branchen fokussieren und damit schwer zu vergleichen sind [8, 10,13, 15-21]. Allerdings fällt auch bei einem ausschließlichen Vergleich der branchenunabhängigen Studien die Spannweite von 19 bis 56 \% ins Auge. Erklären lässt sich diese mit dem unterschiedlich hohen Rücklauf, der bspw. in der Untersuchung von Beck et al. [11] nur 15\% beträgt. Die Vermutung liegt nahe, dass insbesondere Betriebe ohne BGF/BGM zu den Verweigerern zählen (Schweigeverzerrung) - ein Problem, mit dem die überwiegende Anzahl der Studien konfrontiert war. Verringern ließe sich dieser Bias nur durch verpflichtende Untersuchungen (z. B. im Rahmen von repräsentativen Erhebungen, etwa im Rahmen bestehender Überwachungsaufträge staatlicher oder berufsgenossenschaftlicher Arbeitsschutzinstitutionen).

Verständnis von BGF/BGM Wie die jeweilige Formulierung der Fragestellung in den einzelnen Erhebungen zeigt, sind sich Wissenschaft und Praxis der Betrieblichen Gesundheitsförderung in Deutschland nicht darüber einig, welche konkreten betrieblichen Bemühungen im Detail unter die Begriffe Betriebliche Gesundheitsförderung bzw. Betriebliches Gesundheitsmanagement fallen. Ein Teil der Untersuchungen, z. B. [9, 12, 15, 22] arbeitet mit Auswahllisten, die meist Struktur- und Prozesselemente sowie Verhaltensangebote enthalten und ja/nein/weiß-nicht-Antworten vorsehen. Ein weiterer praktizierter Zugang besteht darin, den Adressaten eine Definition vorzulegen und sie dazu aufzufordern, die Antworten auf diese zu beziehen z. B. $[8,13,17,20]$. Ein Problem stellt in diesem Zusammenhang die Frage dar, inwieweit eine Abgrenzung zwischen Gesundheitsförderung und Arbeitsschutz möglich und sinnvoll ist. Angesichts der, durch die europäische Arbeitsschutzgesetzgebung erreichte Annäherung zwischen beiden Bereichen und infolge der durchaus wünschenswerten Integration partizipativer Elemente und psychosozialer Themen in den Arbeitsschutz [5] erscheinen Abgrenzungen in dem Sinne, dass nur Maßnahmen eruiert werden, die über den gesetzlich vorgeschriebenen Arbeitsschutz hinausgehen z. B. [9, 12,17] ebenso problematisch wie der Versuch, arbeitsschutzbezogene Aktivitäten in die Untersuchung generell einzubeziehen z. B. [15, 16, 21, 22]. Der letztere, in einigen Studien verfolgte Ansatz dürfte für die ermittelten, hohen Umsetzungsgrade mitverantwortlich sein. Während die meisten anderen Studien nach der konkreten Umsetzung von BGF/BGM-Elementen fragen, interessierte sich die Studie von Jung et al. [13] für positive Einstellungen im Management zugunsten von BGF/BGM. Die aus diesen Beobachtungen abzuleitende Forderung mündet in das Postulat, in künftigen Untersuchungen eine einheitliche und operationale Definition dessen anzustreben, was im Einzelnen in Zusammenhang mit BGF/BGM erfasst werden soll.

Untersuchungszeitraum Die meisten Studien benennen keinen eindeutigen Zeitpunkt oder Zeitraum, auf den sich die Recherche bezieht, sondern fragen generell, ob BGF/BGM bzw. bestimmte Maßnahmen im Unternehmen realisiert werden. Inwieweit sich die Antworten daher im Sinne einer Punktprävalenz auf den konkreten Erhebungszeitpunkt beziehen oder einen längeren Zeitraum im Auge haben, bleibt dabei oft der Interpretation der jeweiligen Respondenten überlassen. Eine Ausnahme hiervon bildet die Untersuchung von Bechmann et al. [10], die ihre Fragestellung explizit auf den aktuellen Zeitpunkt des Interviews beziehen. In ihrer Längsschnittbetrachtung fragen Groeben et al. nach Veränderungen in der Verbreitung von Maßnahmen der BGF in den letzten 5 bzw. 6 Jahren $[15,16]$, während im Querschnitt danach differenziert 
wurde, ob die Maßnahme „abgeschlossen“, „derzeit erprobt“, „ab und zu durchgeführt“ „Routine“ oder „geplant“ ist [15]. Inwieweit der erfasste Zeitraum in den übrigen Studien einen Einfluss auf die Antworten hatte, kann letztlich nicht geklärt werden. Abgeleitet werden sollte für künftige Analysen jedoch die Forderung nach einer möglichst konkreten Definition des interessierenden Prävalenzzeitraums.

Erhebungszeitpunkt Angesichts der seit 2007 bestehenden Verpflichtung der Krankenkassen zugunsten der Realisierung von BGF/ BGM und einer zunehmenden allgemeinen Informationsdichte zum Thema lässt sich erwarten, dass die betriebliche Akzeptanz entsprechender Aktivitäten in den letzten Jahren gestiegen ist. Ältere Studien müssten nach dieser Annahme geringere Prävalenzen aufweisen als jüngere. Da es sich bei den meisten Arbeiten um Querschnittanalysen handelt, und sich ein studienübergreifender Vergleich als problematisch erweist, lässt sich diese Vermutung lediglich anhand der wenigen vorliegenden Kohorten- bzw. Langzeitstudien prüfen. Entgegen dieser Hypothese verzeichnet die Arbeitsgruppe um Gröben im Zeitraum von 1997 bis 2003 in der Dienstleistungs- und Metallverarbeitungsindustrie einen Rückgang bei der quantitativen Verbreitung [16, 18, 21]; die Autoren betonen aber den parallel dazu erfolgten, leichten Anstieg in der Qualität der Maßnahmen. Eine erhöhte quantitative Akzeptanz stellen dagegen Hollederer et al. auf Basis der wiederholten Auswertungen der IAB-Paneldaten fest. So ist in den Jahren von 2002 über 2004 bis 2012 der Anteil der Betriebe mit BGF von 19\% über $20 \%$ auf $27 \%$ angestiegen [9, 12]. Um die Annahme einer zunehmenden Akzeptanz von BGF/BGM zu bestätigen, wären weitere Langzeit-, insbesondere Kohortenstudien mit einem einheitlichen Design und der Kontrolle von Drop-Out-Fällen vonnöten.

Erfasste Interventionen Die Auseinandersetzung mit den in den Studien erfassten Einzelmaßnahmen zeigt eine erhebliche Heterogenität bei der Erfassung und Zuordnung von Aktivitäten. Während einige Arbeiten bspw. eher strukturelle und strategische Elemente von BGF/BGM wie Steuerkreise und Bedarfsanalysen erheben, konzentrieren sich andere stärker auf konkrete Aktionen und Programme, die unmittelbar auf Beschäftigte ausgerichtet sind. Bestimmte Maßnahmen wurden nur in einzelnen Studien erfasst und entziehen sich damit einer Vergleichbarkeit, z. B. Arbeitszeitgestaltung [20]. Besonders erschwert die unterschiedliche Zuordnung konkreter Maßnahmen zu Themenkomplexen einen allgemeingültigen Überblick. So erfassen Bechmann et al. [10] eine „regelmäßige Analyse der betrieblichen Situation“ ohne diese näher zu bestimmen, während sich andere Erhebungen speziell nach Mitarbeiterbefragungen oder der Auswertung von Arbeitsunfähigkeitsdaten erkundigen. Noch unübersichtlicher ist der Status Quo im Hinblick auf verhaltensbezogene Programme, die in sehr unterschiedlicher Weise kategorisiert werden. Während Hollederer [12] z. B. Mitarbeiterfortbildungen, Seminare, Lehrgänge, Schulungen, Erste-Hilfe-Kurse u. ä. als Einheit erfasst und mit $5 \%$ beziffert, differenzieren Ulmer and Groeben [21] nach Kommunikationstrainings (28\%), Managementseminaren (27\%), KonfliktmanagementSeminaren (27\%), Seminaren zur Teamzusammenarbeit (21\%). Andere Angaben wie „Verbesserung der sozialen Beziehungen am Arbeitsplatz“ [17] lassen offen, um welche Art von Intervention es sich konkret handelt. Aufgrund dieser Divergenzen war ein Rangsummenvergleich nicht möglich, sodass die Frage, welche Maßnah- men generell am häufigsten umgesetzt werden, auf Basis der gegebenen Datenlage nicht zu beantworten ist. Eine Prioritätensetzung zugunsten verhaltenspräventiver Aktivitäten lässt sich auf Grundlage der vorliegenden Studien für die betrieblicherseits beschriebenen Programme nicht bestätigen. Wichtig für die künftige Forschung wäre eine Einigung auf ein gemeinsames Erfassungsund Kategorisierungsschema, anhand dessen valide und vergleichbare Angaben möglich sind.

\section{Ergebnisdiskussion}

Die hier zusammengestellten Erhebungen basieren zum Teil auf umfangreichen und aufwändigen Untersuchungen und liefern wertvolle Beiträge zur Erhöhung der Transparenz über den Zustand von BGF/BGM in Deutschland. Allerdings fußen die Arbeiten auf divergierenden Designs, Stichprobenauswahlen und Fragestellungen und kommen demzufolge zu heterogenen Ergebnissen. Zudem erweist es sich immer wieder als schwierig, Aktivitäten der BGF/des BGM adäquat zu erfassen: Neben der Komplexität des Konzepts und der Vielfalt der darunter subsumierten Interventionen erschweren mehrdeutige Begriffsverständnisse und Zugänge eindeutige Aussagen über die Verbreitung von BGF/BGM.

Einen weiteren kritischen Punkt stellt die Identifikation möglicher Einflussfaktoren auf die Wahrscheinlichkeit der Umsetzung von BGF dar. Angesichts des Umstandes, dass es sich bei den meisten der vorgestellten Arbeiten um Querschnittanalysen handelt, ist die Frage nach der Kausalität an einigen Stellen nicht eindeutig zu beantworten. Ob bspw. das Vorhandensein eines Gesundheitskoordinators [11], die Zahl der Hierarchieebenen und der Dauerarbeitsverhältnisse [13] Ursache oder Folge für die Umsetzung von BGF/BGM darstellen, oder welches die faktischen Ursachen einer höheren Verbreitung in den neuen Bundesländern sind, müsste im Rahmen weiterer Analysen geklärt werden. Auch für weitere Merkmale besteht Unklarheit hinsichtlich der Kausalität der Zusammenhänge, die im Rahmen weiterer Studien geklärt werden sollten. So identifizieren Köhler et al. [17] sowie Beck et al. [11] wirtschaftlichen Druck als begünstigenden Einflussfaktor, während Jung et al. [13] dem wirtschaftlichen Erfolg eine positive Wirkung zuschreiben. Unter welchen Bedingungen sich die wirtschaftliche Situation in welcher Weise auf die Akzeptanz von BGF/BGM auswirkt, dürfte von weiteren, intervenierenden Größen abhängen, die in künftigen Studien zu ermitteln wären.

\section{Weitergehende Überlegungen zur Erfassbarkeit der Wirkung von BGF}

In der vorliegenden Analyse wurden quantitativ angelegte Arbeiten ausgewertet, die sich typischerweise auf eine Kategorisierung und Digitalisierung bzw. Quantifizierung der erfassten Phänomene stützen. Was diese Analysen kaum zu erfassen vermögen, ist die Qualität des betrieblichen Miteinanders und der Organisationskultur, der Arbeitsbedingungen, des Führungsverhaltens und der erlebten Gratifikation und Wertschätzung. Wie die arbeitswissenschaftliche Forschung der letzten Jahre sehr valide nachweisen konnte, sind es vor allem diese Merkmale, die über Wohlbefinden und Gesundheit von Beschäftigten entscheiden [23-26]. Gesundheitsfördernde betriebliche Aktivitäten müssen sich deshalb daran messen lassen, inwieweit sie in der Lage sind, diese Bedingungen 
herzustellen oder zumindest eine Annäherung herbeizuführen. Betriebliche Bedarfsanalysen bspw. können nur dann eine positive Wirkung entfalten, wenn sie dazu dienen, Veränderungsnotwendigkeiten aus Sicht der Beschäftigten zu identifizieren, und wenn ernsthaft und ehrlich an den daraus resultierenden Themen gearbeitet wird. Selbst partizipative Maßnahmen wie Gesundheitszirkel sind nur so wertvoll, wie die seitens der Beschäftigten ausgearbeiteten Anregungen von den Entscheidungsverantwortlichen aufgegriffen und umgesetzt werden. Gleichfalls sind die vonseiten managementorientierter Ansätze besonders hervorgehobenen Struktur- und Prozesselemente - etwa die Formulierung strategischer Ziele und Planungen, die Einrichtung von Steuerungskreisen u. a. m. z. B. [27, 28] kein Selbstzweck. Vielmehr haben sie die Aufgabe, Bedingungen herzustellen, die „Arbeit zu einer Quelle der Gesundheit und nicht der Krankheit" machen [29]. Die Qualität von BGF/BGM besteht demnach nicht nur im schematischen Vollzug von Prozessen, sondern zeigt sich darin, wie die ermittelten Strukturen, Prozesse und Maßnahmen im Einzelnen ausgestaltet werden - etwa in der Glaubwürdigkeit ihrer Versprechungen, im Ausmaß wertschätzender betrieblicher Kommunikation oder darin, wie Mitarbeiter als Subjekte wahrgenommen, gefördert, unterstützt und gefordert werden. Wenn es gelingt, den Fokus der Forschung nicht nur auf das „Was“ zu richten, sondern auf das „Wie“ kommen wir der Frage näher, ob BGF/BGM einen Beitrag zu mehr Gesundheit bei der Arbeit leisten kann. Die quantitative Erfassung von Maßnahmen ist nur ein erster Schritt innerhalb eines solchen Forschungsportfolios.

\section{FAZIT}

Bezüglich der Umsetzung von Betrieblicher Gesundheitsförderung in der deutschen Wirtschaft liegen heute diverse Untersuchungen vor, die allerdings hinsichtlich Fragestellung, Repräsentativität und Erhebungsdesign - und damit auch im Ergebnis wesentlich unterscheiden. Generelle Aussagen hinsichtlich der Umsetzung von BGF/BGM sind daher schwierig zu treffen. Dennoch lassen sich spezifische Merkmale benennen, die in einem Großteil der Studien übereinstimmend als begünstigende Einflussfaktoren identifiziert wurden. Dazu scheinen vor allem die Betriebsgröße und das Vorhandensein einer Beschäftigtenvertretung zu gehören. Für die in Einzelstudien gefundenen, zahlreichen weiteren Merkmale sind weitergehende Einzelstudien ebenso wie Metaanalysen nötig. Um den Erfolg der BGF/BGM-Aktivitäten adäquat erfassen zu können, empfehlen sich neben quantitativen Analysen Triangulationen aus quantitativen und qualitativen Designs.

\section{Danksagung}

Vielen Dank für die hilfreichen Anregungen und Hinweise zur Überarbeitung des Manuskripts.

\section{Interessenkonflikt}

Die Autorin gibt an, dass kein Interessenkonflikt besteht.
Literatur

[1] MDS. Präventionsbericht 2015. Leistungen der gesetzlichen Krankenversicherung: Primärprävention und betriebliche Gesundheitsförderung: Berichtsjahr 2014. Berlin 2015;

[2] Badura B, Schröder H, Vetter C. Fehlzeiten-Report 2008. Betriebliches Gesundheitsmanagement: Kosten und Nutzen. Heidelberg, New York: Springer; 2009

[3] Bräunig D, Haupt J, Kohstall T et al. Wirksamkeit und Nutzen betrieblicher Prävention. iga-Report 28. Berlin 2015;

[4] Eberle G. Erfolgsfaktor Betriebliches Gesundheitsmanagement - betriebswirtschaftlicher Nutzen aus Unternehmersicht. Prävention. In: Kirch W, Badura B. (ed.). Prävention. Berlin, Heidelberg: Springer; 2006: 325-338

[5] Faller G, Faber U. Hat BGF eine rechtliche Grundlage? Gesetzliche Anknüpfungspunkte für die Betriebliche Gesundheitsförderung in Deutschland. In: Faller G, (Hrsg.). Lehrbuch Betriebliche Gesundheitsförderung. Bern: Huber; 2012: 9-52

[6] ENWHP. Die Luxemburger Deklaration zur betrieblichen Gesundheitsförderung in der Europäischen Union; 2007. Im Internet http://www. luxemburger-deklaration.de Stand: 15 September 2015;

[7] Badura B, Ritter W, Scherf M. Betriebliches Gesundheitsmanagement - ein Leitfaden für die Praxis. Berlin: sigma; 1999

[8] Ansmann L, Jung J, Nitzsche A et al. Zusammenhänge zwischen der Betriebsstruktur und Betrieblichem Gesundheitsmanagement in der Informationstechnologie- und Kommunikationsbranche. Gesundheitswesen 2012; 74: 298-305

[9] Hollederer A, Wießner F. Prevalence and development of workplace health promotion in Germany: results of the IAB Establishment Panel 2012. Int Arch Occup Environ Health 2014 published online

[10] Bechmann S, Jäckle R, Lück $P$ et al. Motive und Hemmnisse für Betriebliches Gesundheitsmanagement (BGM). iga Report 20. Berlin, Essen 2010;

[11] Beck D, Lenhardt U, Schmitt B et al. Patterns and predictors of workplace health promotion: cross-sectional findings from a company survey in Germany. BMC Public Health 2015; 15: $343 f f$

[12] Hollederer A. Betriebliche Gesundheitsförderung in Deutschland - Ergebnisse des ABBetriebspanels 2002 und 2004. Gesundheitswesen 2007; 69: 63-76

[13] Jung N, Nitzsche A, Ansmann L et al. Organizational factors and the attitude toward health promotion in German ICT-companies. Health Promotion International 2012; 27: 382-393

[14] Zelfel RC, Alles T, Weber A. Gesundheitsmanagement in kleinen und mittleren Unternehmen - Ergebnisse einer repräsentativen Unternehmensbefragung. Das Gesundheitswesen 2011; 73: 515-519

[15] Gröben F, Wenninger S. Betriebliche Gesundheitsförderung im öffentlichen Dienst. Ergebnisse einer Wiederholungsbefragung von Führungskräften in Hessen und Thüringen. Prävention und Gesundheitsförderung 2006; 2: 94-98

[16] Gröben F, Ulmer ]. Gesundheitsförderung im Betrieb. Düsseldorf: Hans-Böckler-Stiftung; 2004

[17] Köhler T, Janssen C, Plath SC et al. Determinants of workplace health promotion in the insurance sector: results of a complete survey of German insurance companies in 2006. Gesundheitswesen 2009; 71: 722-731

[18] Gröben F. Betriebliche Gesundheitsförderung in Hessen und Thüringen. Ergebnisse einer Betriebsbefragung. Prävention 2000; 23: 17-21

[19] Gröben F. Ergebnisse einer Umfrage bei Führungskräften zur Prävention und betrieblichen Gesundheitsförderung im öffentlichen Dienst in Hessen und Thüringen. In: Badura B, Litsch M, Vetter C, (Hrsg.). Fehlzeitenreport 2001: Gesundheitsmanagement im öffentlichen Sektor. Berlin, Heidelberg: Springer; 2002: 50-62 
[20] Plath SC, Köhler T, Krause H et al. Prevention, health promotion and workplace health management in German banks: Results from a nationwide representative survey. Journal of Public Health 2008; 16 : 195-203

[21] Ulmer J, Groeben F. Work place health promotion. A longitudinal study in companies placed in Hessen und Thueringen. Journal of Public Health 2005; 13: 144-152

[22] Wartmann A, Reinheckel A. Ist betriebliche Gesundheitsförderung in Kleinbetrieben ein Thema? Prävention 2006; 29: 47-49

[23] Badura B, Hehlmann T. Betriebliche Gesundheitspolitik. Berlin, Heidelberg, New York: Springer; 2003

[24] Fuchs M. Sozialkapital: nicht nur produktiv, sondern auch gesund. In: Faller G, (Hrsg.). Lehrbuch Betriebliche Gesundheitsförderung. Bern: Huber; 2012: 113-121
[25] Moldaschl M. Das Konzept der Widersprüchlichen Arbeitsanforderungen (WAA). Ein nichtlinearer Ansatz zur Analyse von Belastung und Bewältigung in der Arbeit. In: Faller G, (Hrsg.). Lehrbuch Betriebliche Gesundheitsförderung. Bern: Huber; 2012: 102-112

[26] Peter R. Von Handlungs- und Entscheidungsspielräumen, Belohnungen und betrieblicher Gerechtigkeit: Die Modelle Demand-Control und berufliche Gratifikationskrise. In: Faller G, (Hrsg.). Lehrbuch Betriebliche Gesundheitsförderung. Bern: Huber; 2012: 79-88

[27] Kaminski M. Betriebliches Gesundheitsmanagement für die Praxis. Wiesbaden: Springer, Gabler; 2013

[28] EuPD Research. Corporate Health Jahrbuch. Bonn 2014;

[29] WHO. The Ottawa Charter for Health Promotion. 1986; Im Internet: http://www.euro.who.int/de/publications/policy-documents/ ottawa-charter-for-health-promotion Stand: 15. September 2015; 